\title{
Rat lungworm Angiostrongylus cantonensis (Chen, 1935) (Nematoda: Strongylida: Metastrongylida) ${ }^{1}$
}

\author{
John Capinera and Heather S. Walden²
}

\section{Introduction and Distribution}

Like many pest and disease problems, rat lungworm (Angiostrongylus cantonensis) has been slowly spreading around the world. First described by Chen (1935) from rats in China, the medical significance of this parasite was overlooked until 1944 when it was found infecting humans in Taiwan. Even then, because the report was published in Japanese, its importance remained largely unknown. In 1955, Mackerras and Sandars found this nematode among rats in Brisbane, Australia, and described its life cycle, including the importance of its molluscan intermediate hosts.

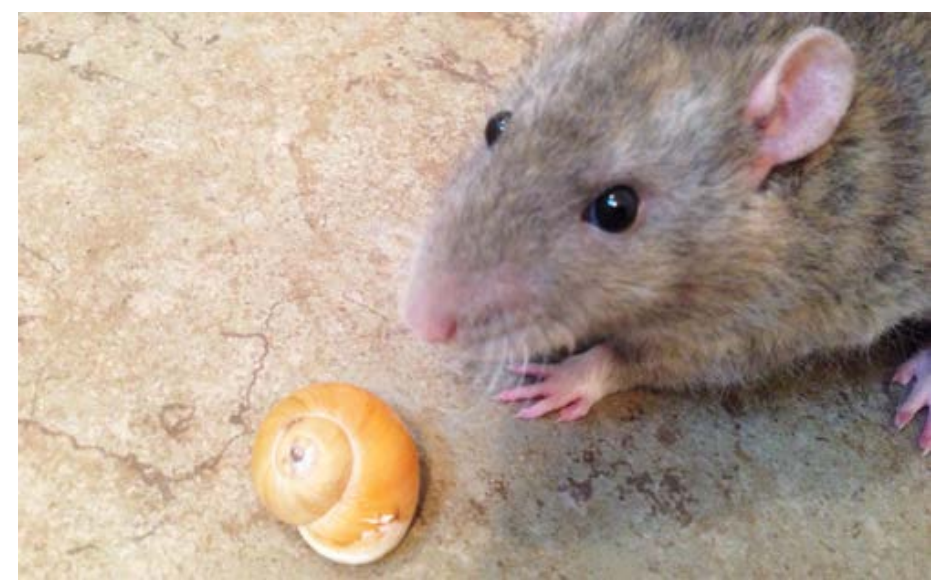

Figure 1. Rats are the normal host of Angiostrogylus cantonensis nematodes. They acquire the parasite by feeding on infected molluscs. Credits: Jennifer L. Gillett-Kaufman
It was found in Hawaii in 1960, and Tahiti in 1961. It has since been detected in many regions of the world, including Cuba (1981), Puerto Rico (1986), New Orleans (1988), and Florida (2003). Rats are the definitive hosts, and the only hosts in which the adult stage of the nematode occurs. However, third-stage larvae are infective to humans if they are accidentally consumed. Ingested worms migrate to the central nervous system of humans and cause eosinophilic meningitis, which often is manifested by fever, headache, stiff neck, nausea, vomiting, fatigue, body ache, skin irritations, and photophobia (Slom et al. 2002). The signs and symptoms may persist for weeks or months.

Although infected humans usually recover, the nematodes can penetrate the brain, spinal cord, and eyes, and sometimes cause paralysis, blindness, or death. Other primates, dogs, horses, and other animals also are susceptible to infection. A particularly good host of the nematode is giant African land snail, Achatina fulica, which has been deliberately relocated to some areas, but movement in association with other molluscs and rats is implicated in the spread of Angiostrongylus cantonensis (Duffy et al. 2004).

\section{Life Cycle}

The life cycle was described by Mackerras and Sandars (1955), and a synopsis follows. When an infected mollusc is consumed by a rat, the third-stage nematode larvae within the mollusc are released in the rat's stomach. From

1. This document is EENY570, one of a series of the Entomology and Nematology Department, UF/IFAS Extension. Original publication date September 2013. Visit the EDIS website at http://edis.ifas.ufl.edu.

2. John L. Capinera, professor/chairman, Department of Entomology and Nematology, University of Florida, Gainesville, FL 32611; Heather S. Walden, research assistant professor, VM - Infectious Diseases and Pathology, UF/IFAS Extension, Gainesville, FL 32611. 
the stomach, the nematode larvae quickly move down the intestine, where they move through the walls of the small intestine into the blood, then enter the lungs and kidneys. Within 24 hours, some nematodes are carried passively to the brain and spinal cord, although many seemingly are lost. These third-stage larvae reaching the brain become sluggish as they absorb food, and they molt to the fourth stage after about 7 days. Thereafter, the fourth-stage larvae are found within the brain and on its surface for about 12 days, then molt to the adult form. The adults continue to scar the surface of the cerebrum and other sections of the brain, depending on the infestation level. Heavy infestation of nematodes can result in significant lesions, changes in behavior of the rat, and even death. Within a month, the nematodes leave the brain and enter the blood, where sexual maturation occurs in about 10 days. Fertilization occurs shortly thereafter, followed by production of eggs, which are released into the blood. The eggs lodge in the smaller vessels of the lungs and hatch, then the first-stage nematodes break into the respiratory tract, migrate to the trachea, and are swallowed. So 40 to 50 days after ingestion, first stage larvae are being released in the rat's feces.

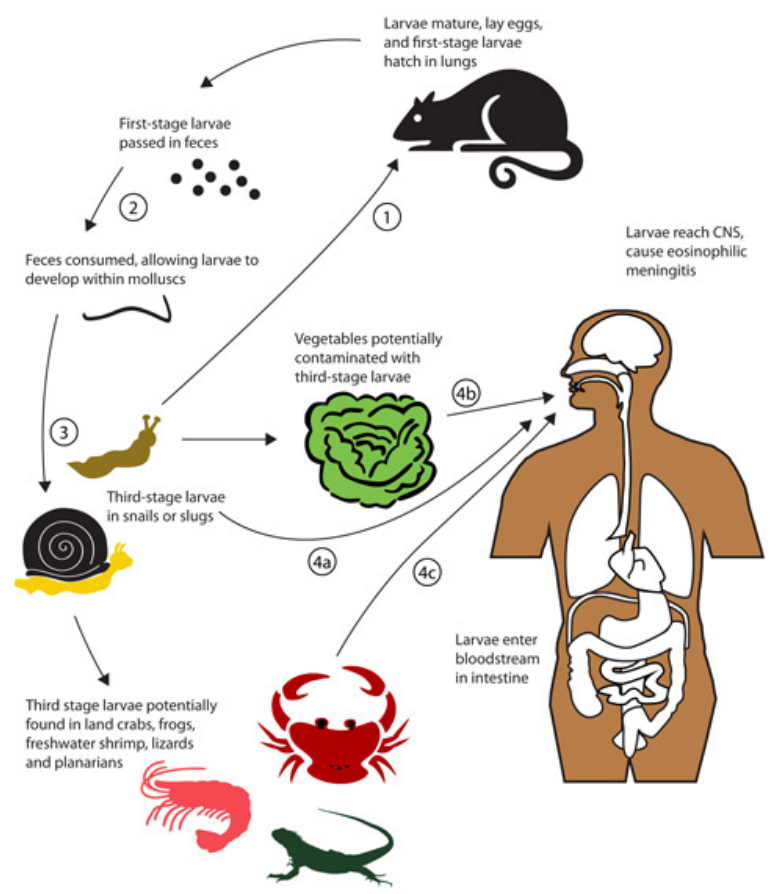

Figure 2. Potential routes of infection of the human central nervous system (CNS) by the rat lungworm, Angiostrongylus cantonensis. Note that the normal life cycle involves (1) consumption of molluscs by rats, then (2) excretion of nematodes in rat feces, which are then (3) consumed by molluscs. Human infection can occur when uncooked infected molluscs are eaten (4a) or, more rarely, when uncooked contaminated paratenic (transport) hosts (4C) or vegetable matter (4b) is consumed. Adapted from Wang et al. (2008).
Rat feces containing first-stage nematode larvae are potentially consumed by snails and slugs, with the young larvae soon penetrating into the molluscs and gaining considerable weight as they absorb food. The nematode larvae soon molt to the second and then third stage in the mollusc host. Nematodes can remain relatively quiescent in the third stage for long periods while awaiting a rat to consume the host molluscs, liberate the nematodes from their old cast skins, and allow them to continue to maturity in another rat.

\section{Epidemiology}

Angiostrongyliasis is acquired by humans when they unintentionally ingest third-stage nematode larvae in food items that are eaten raw, or undercooked. Angiostrongyliasis refers basically to eosinophilic meningitis. Eosinophils are a type of disease-fighting white blood cells, and their concentration in the blood increases when foreign organisms such as parasites are present. Meningitis is an inflammation of the meninges, a protective covering of the brain and spinal cord, and nematodes can induce such inflammation in humans and other primates. Often the contaminated food item is some type of terrestrial mollusc (snail or slug). A survey conducted in China of potential mollusc sources of Angiostrongyliaisis indicated that large, nonindigenous species such as Achatina fulica and Pomacea canaliculata destined for human consumption were sometimes infected (Lv et al. 2009a). Additionally, infection sometimes results from consumption of fresh-water shrimp, land crabs, or predatory planaria, and possibly from frogs and lizards (Alicata 1991, Wang et al. 2008, Herwaldt 2012). Uncooked vegetables contaminated by molluscs are also a potential route of infection, as several incidents of human infection due to ingestion of vegetables or vegetable juice are reported (Cross 1987, Wang et al. 2008). These alternate routes of infection are shown in Figure 1. It does not appear that the nematodes can be secreted in the slug mucus (Qvarnstrom et al. 2007); however, Heyneman and Lim (1967) reported Angiostrongylus cantonensis third stage larvae were shed in the mucus of the Malayan slug, Microparmarion malayanus. The incubation period of this disease is highly variable, ranging from 1 day to 7 months, depending largely on the number of parasites ingested, but symptoms often are expressed within 2 weeks.

Molluscs are intermediate hosts (these hosts are required for part of the nematode's development; they are an essential part of the life cycle), whereas shrimp, crabs, frogs, lizards, and planaria are paratenic (transport) hosts (they are accidental carriers, and not required for successful development of the nematode, but assist in movement 
of the nematodes). Most terrestrial molluscs, and some aquatic molluscs, seem to be susceptible to infection (Cross 1987). Among the snails and slugs found in North America that have been shown to support Angiostrogylus cantonensis are Euglandina rosea, Mesodon thyroidus, Bradybaena similaris, Anguispira alternata, Subulina octona, Polygyra triodontoides, Belocaulus angustipes (= Vaginulus ameghini), Philomycus carolinianus, Deroceras laeve, Limax flavus, and Lehmannia valentiana (= L. poirieri) (Campbell and Little 1988), Phyllocaulis variegatus (Maldonado et al. 2012), Achatina fulica (Cross 1987), Pomacea canaliculata (Wang et al. 2008), and Zachrysia provisoria (unpublished). The smaller molluscs tend to support fewer nematodes (Campbell and Little 1988), and thus are less likely to be a problem, as the severity of the problem is related to how many nematodes are ingested (Maldonado et al. 2012).

The role of vertebrate animals in the epidemiology of Angiostrongyliasis is variable. Rats probably are necessary for establishment of Angiostrogylus cantonensis. Rats are ubiquitous, but much more numerous in some environments. Rattus rattus and Rattus norvegicus are the rats normally implicated in disease transmission, but other rats are suitable hosts. Frogs and lizards are thought to have a minor role as transport hosts, but they should be cooked thoroughly before consumption. Primates, including humans, are not normal hosts, and are only rarely infected. When primates are infected, their response is quite variable, ranging from the mild symptoms (fever, headache, etc.), to eosinophilic meningitis or even death. The nematodes reaching the brain of primates cannot continue further, but die and initiate eosinophilic meningitis. Although primates usually survive infection, captive primates and humans in several countries have been reported to experience lethal infection. Massive exposure to infective third-stage larvae seems to be required for mortality of the host.

Diaz (2010) suggests that frequent travelers are especially at risk due to their behavior, including: willingness to eat exotic and unfamiliar food that might be raw or undercooked; relaxation of normal sanitary habits while traveling, such as hand washing; lack of familiarity of reservoirs of infectious diseases; and preference for wilderness, tropical, and beach resorts that might be especially likely to harbor rodents, molluscs, and Angiostrogylus cantonensis.

\section{Morphology}

Adults are filiform in both sexes, tapering at the anterior end (Fig. 2). Females are larger and more robust. The adult male nematodes are $15.5-23.0 \mathrm{~mm}$ long and $0.25-0.35 \mathrm{~mm}$ wide. They are transparent, with a smoothly rounded head.
Two or three minute triangular teeth are located at the base of the oral cavity. The esophagous is $0.29-0.35 \mathrm{~mm}$ long. The intestine is wide but thin-walled. The excretory pore located in the region of the esophageal-intestinal junction. Females are larger, measuring 18.5-33.0 $\mathrm{mm}$ in length and 0.28-0.5 mm wide. The head, esophagous, and intestine are similar to the male. The ovaries fill the posterior region. The vulva is a transverse slit located about $0.25 \mathrm{~mm}$ from the posterior end.

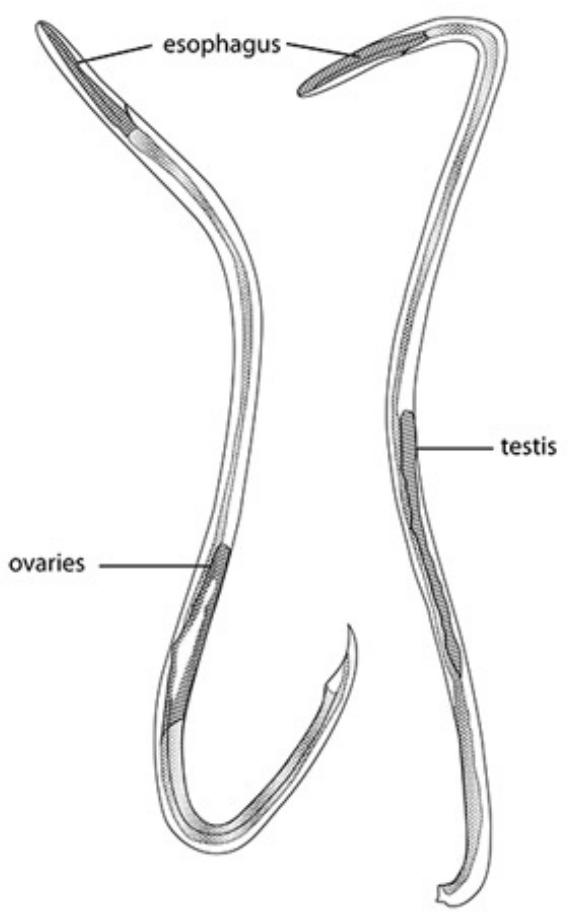

Figure 3. Adult female (left) and male (right) of the rat lungworm, Angiostrongylus cantonensis. Adapted from Mackerras and Sandars (1955).

The juvenile nematodes are much smaller. The first-stage larva is only $0.27-0.30 \mathrm{~mm}$ in length and has a distinct notch on the tail, the second measures $0.42-0.47 \mathrm{~mm}$, and the third stage $0.42-0.49 \mathrm{~mm}$. During the latter portion of the second stage the larva, which is still enclosed in the first cast skin, molts again, and is now enclosed in two cast skins. The third-stage larva remains within the two cast skins until liberated by digestion in the rat's stomach. The fourth-stage larva, which is found in rats but not intermediate hosts, is $0.85-1.0 \mathrm{~mm}$ long and has a more pointed tail than earlier stages. A detailed description of each stage is provided by Mackerras and Sandars (1955); additional useful information on morphology is provided by Lv et al. (2009b) and Maldonado Jr. et al. (2012).

\section{Management}

Once Angiostrongylus cantonensis becomes established in populations of rats, it is likely to be a permanent concern. Eradication of rats and molluscs potentially could eliminate 
the problem, but this is impossible to accomplish. Their abundance can be reduced, however, minimizing risk of infection.

Public education can go a long way toward reducing the probability of human infection. Consumption of raw or undercooked terrestrial molluscs should be discouraged both by humans and captive primates. Similarly, paratenic hosts should not be consumed without cooking. The importance of raw vegetable or vegetable juice consumption as a route of infection is more questionable. Although it is possible for vegetables to be contaminated by some species of molluscs containing infective third-stage juvenile nematodes, it is rare and seems improbable if normal sanitary practices are followed. Commercially produced vegetables should have little likelihood of being contaminated, as the soil is tilled, weeds are suppressed, and pesticides are applied as needed.

However, if snails or slugs become abundant in home garden or other vegetable production areas and the produce is to be eaten raw, the produce should be examined for molluscs and washed thoroughly. Soaking vegetables with $1.5 \%$ bleach solution for 15 minutes (4 tablespoons or $2 \mathrm{oz}$ of bleach per gallon of rinse water; or $15 \mathrm{ml}$ of bleach per liter of water) has been shown to be effective for control of Angiostrongylus costaricensis (Zanini and Graeff-Teixeira 2001), and this decontamination procedure might be advisable for leafy vegetables that are potentially contaminated by Angiostrongylus cantonensis as well.

\section{Selected References}

Alicata JE. 1991. The discovery of Angiostrongylus cantonensis as a cause of human eosinophilic meningitis. Parasitology Today 7: 151-153.

Campbell BG, Little MD. 1988. The finding of Angiostrongylus cantonesis in rats in New Orleans. American Journal of Tropical Medicine and Hygiene 38: 568-573.

Cross JH. 1987. Public health importance of Angiostrongylus cantonensis and its relatives. Parasitology Today 3: 367-369.

Diaz JH. 2010. Recently reemerging helminthic infections causing eosinophilic meningoencephalitis: neuroangiostrogyliasis, baylisascariasis, and gnathostomiasis. Journal of Neuroparasitology 1: 1-14.

Duffy MS, Miller CL, Kinsella JM, de Lahunta A. 2004. Parastrongylus cantonensis in a nonhuman primate, Florida. Emerging Infectious Diseases 10:2207-2210.
Herwaldt BL. 2012. Angiostrogyliasis (Angiostrongylus cantonensis infection, neurologic angiostrongyliasis). Centers for Disease Control and Prevention, Yellowbook, chapter 3 .

Heyneman D, Lim B. 1967. Angiostrongylus cantonensis: proof of direct transmission with its epidemiological implications. Science 158: 1057-1058.

Lv S, Zhang Y, Liu H-X, Hu L, Yang K, Steinmann P, Chen Z, Wang L-Y, Utzinger J, Zhou S-N. 2009a. Invasive snails and an emerging infectious disease: results from the first national survey on Angiostrongylus cantonensis in China. PLOS Neglected Tropical Diseases 3(2): e368.

Lv S, Zhang Y, Liu H-X, Zhang C-W, Steinmann P, Zhou S-N, Utsinger J. 2009b. Angiostrongylus cantonensis: morphological and behavioral investigation within the freshwater snail Pomacea canaliculata. Parasitology Research 104: 1351-1359.

Mackerras MJ, Sandars DF. 1955. The life history of the rat lung-worm, Angiostrongylus cantonensis (Chen) (Nematoda: Metastrongylidae). Australian Journal of Zoology 3: $1-25$.

Maldonado Jr. A, Simões R, Thiengo S. 2012. Angiostrongyliasis in the Americas, chapter 17, In Zoonosis, LornezoMorales J (ed.). InTech, Rijeka, Croatia. DOI 10.5772/38632

Slom TJ, Cortese MM, Gerber SI, Jones RC, Holtz TH, Lopez MHS, Zambrano CH, Sufit RL, Sakilvaree Y, Chaicumpa W, Herwaldt BL, Johnson S. 2002. An outbreak of eosinophilic meningitis caused by Angiostrongylus cantonensis in travelers returning from the Caribbean. New England Journal of Medicine 346: 668-574.

Qvarnstrom Y, Sullivan JJ, Bishop HS, Hollingsworth R, da Silva, AJ. 2007. PCR-based detection of Angiostrongylus cantonensis in tissue and mucus secretions from molluscan hosts. Applied and Environmental Microbiology 73: 1415-1419.

Wang, Q-P, Lai D-H, Zhu XQ, Chen Z-G, Lunn Z-R. 2008. Human angiostrongyliasis. Lancet Infectious Diseases 8: 621-630.

Zanini GM, Graeff-Teixeira C. 2001. Inactivation of infective larvae of Angiostrongylus costaricensis with short time incubations in $1.5 \%$ bleach solution, vinegar or saturated cooking salt solution. Acta Tropica 78: 17-21. 